\title{
Transverse Scattering and Generalized Kerker Effects in All-Dielectric Mie-Resonant Metaoptics
}

\author{
Hadi K. Shamkhi, ${ }^{1}$ Kseniia V. Baryshnikova, ${ }^{1}$ Andrey Sayanskiy, ${ }_{1}^{1}$ Polina Kapitanova, ${ }^{1}$ Pavel D. Terekhov, ${ }^{1,2}$ Pavel Belov, ${ }^{1}$ \\ Alina Karabchevsky, ${ }^{1,2}$ Andrey B. Evlyukhin, ${ }^{3}$ Yuri Kivshar, ${ }^{1,4}$ and Alexander S. Shalin ${ }^{1}$ \\ ${ }^{1}$ ITMO University, St. Petersburg 197101, Russia \\ ${ }^{2}$ Electrooptics and Photonics Engineering Department, School of Electric Engineering, \\ Ben-Gurion University, Beer-Sheva 8410501, Israel \\ ${ }^{3}$ Institute of Quantum Optics, Leibniz, University, Hannover 30167, Germany \\ ${ }^{4}$ Nonlinear Physics Center, Australian National University, Canberra Australian Capital Territory 2601, Australia
}

(Received 6 September 2018; published 17 May 2019)

\begin{abstract}
All-dielectric resonant nanophotonics lies at the heart of modern optics and nanotechnology due to the unique possibilities to control scattering of light from high-index dielectric nanoparticles and metasurfaces. One of the important concepts of dielectric Mie-resonant nanophotonics is associated with the Kerker effect that drives the unidirectional scattering of light from nanoantennas and Huygens metasurfaces. Here we suggest and demonstrate experimentally a novel effect manifested in the nearly complete simultaneous suppression of both forward and backward scattered fields. This effect is governed by the Fano resonance of an electric dipole and off-resonant quadrupoles, providing necessary phases and amplitudes of the scattered fields to achieve the transverse scattering. We extend this concept to dielectric metasurfaces that demonstrate zero reflection with transverse scattering and strong field enhancement for resonant light filtering, nonlinear effects, and sensing.
\end{abstract}

DOI: 10.1103/PhysRevLett.122.193905

Introduction.- - Light scattering by subwavelength particles is closely associated with the Mie resonances and optically induced multipolar response [1,2]. The coexistence and interplay of electric and magnetic dipolar modes make it possible to achieve either constructive or destructive interference leading to a remarkable variety of scattering patterns of subwavelength dielectric particles $[3,4]$. In particular, strong forward-to-backward asymmetric scattering (often termed the Kerker effect) is achieved as a result of interfering electric and magnetic dipole modes [5-9] or quadrupole modes with appropriate phase relations $[4,10,11]$. The Kerker effect is also of great interest to the near-field directionality of Huygens dipoles [12], and it drives the physics of highly efficient Mie-resonant passive and active dielectric metasurfaces and metadevices [13-17].

Overlapping the electric and magnetic multipolar resonances of higher orders opens the way for novel strategies in the effective shaping of light beyond the conventional forward and backward directions [4]. For example, an isolated $V$-shaped plasmonic nanoantenna [18] or a nanoparticle trimer [19] were suggested to achieve side-directed scattering by breaking the scatterer's symmetry. Moreover, the specific conditions for the simultaneous cancellation of both forward and backward scattering were obtained in the quasistatic approximation for radially anisotropic particles [20]. However, in order to satisfy the power conservation and suppress the forward scattering at the same time, the suggested particles should possess a gain.
Recently, the scattering of three-layer onionlike nanoparticles was optimized to achieve anisotropic elliptical side-scattering patterns and suppress both forward and backward scattering [21]. However, the described scenario appears to be not generic, and the layered geometry looks too complicated and not feasible.

Here we predict and demonstrate a new general effect in high-index resonant metaoptics characterized by transverse isotropic scattering by subwavelength nanoparticles with simultaneous suppression of both forward and backward scattered fields. We consider the overlapping of four multipoles (two dipoles and two quadrupoles, see Fig. 1) and reveal the physical mechanisms governing the transverse scattering, providing the required phase and amplitude matching of the scattered fields. We carry out the proofof-principle microwave experiment (being possible due to the scalability of the dielectric particles) and demonstrate a nearly perfect agreement with our analytical results based on Mie theory [22] and our full-scale numerical calculations [23]. Moreover, we design nearly invisible metasurfaces with zero reflection and near 100\% transmission governed by the simultaneous cancellation of both forward and backward scattering in each meta-atom. The transverse scattering is accompanied by a strong near-field localization inside the particles, and it is conceptually similar to the optical anapoles $[24,25]$. Such interference-driven effects are in high demand for various applications, including four-wave mixing [26] and enhanced second-harmonic generation [27]. 


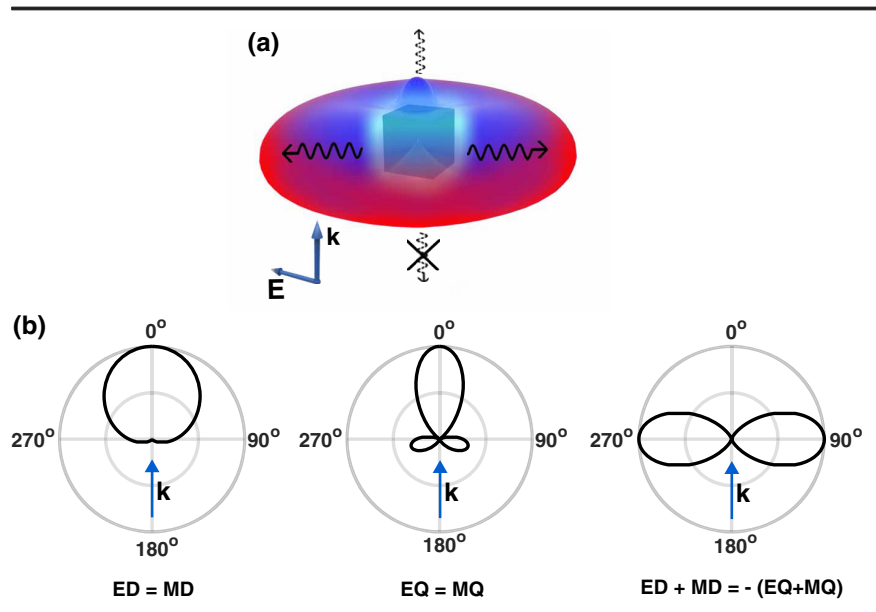

FIG. 1. (a) Transverse scattering pattern for a cubic nanoparticle. Small forward scattering corresponds to a contribution of higherorder multipoles in accordance with the optical theorem. (b) Concept of the formation of an ideal transverse scattering pattern. Electric dipole (ED) is in phase with a magnetic dipole (MD), and an electric quadrupole (EQ) is in phase with a magnetic quadrupole (MQ), whereas the dipoles are out of phase with the quadrupoles.

Concept.-We start with the Cartesian multipole decomposition of the field scattered by an arbitrary subwavelength particle. The surrounding medium is a free space with relative permittivity $\varepsilon=1$. The incident wave is assumed to be linearly polarized along the $x$ axis, $\mathbf{E}_{\text {inc }}=E_{0} e^{i k z} \mathbf{x}$, where $k=|\mathbf{k}|$ is the wave number and $\mathbf{x}$ is a unit vector along the $x$ axis. The scattered light is defined by a superposition of multipoles (up to the quadrupole terms) [28],

$$
\begin{aligned}
\mathbf{E}_{\mathrm{sca}}(\mathbf{n}) \cong & \frac{k^{2} e^{i \mathbf{k} \cdot \mathbf{r}}}{4 \pi r \varepsilon_{0}}\left([\mathbf{n} \times[\mathbf{p} \times \mathbf{n}]]+\frac{1}{c}[\mathbf{m} \times \mathbf{n}]\right. \\
& \left.+\frac{i k}{6}[\mathbf{n} \times[\mathbf{n} \times(\hat{Q} \cdot \mathbf{n})]]+\frac{i k}{2 c}[\mathbf{n} \times(\hat{M} \cdot \mathbf{n})]\right),
\end{aligned}
$$

where $\mathbf{n}=\mathbf{r} / r$ is the unit vector directed from the particle's center towards an observation point, $c$ is the speed of light, and $\mathbf{p}(\mathbf{m})$ and $\hat{Q}(\hat{M})$ are the electric (magnetic) dipole and electric (magnetic) quadrupoles, respectively. Below, we consider two different cases: spherical particles described by the Mie theory, for which Eq. (1) can be simplified in terms of polarizabilities, and nonspherical particles described by the set of tensors from Eq. (1). For spherical particles, we can define the electric and magnetic dipolar $\left(\alpha_{p}, \alpha_{m}\right)$ and quadrupolar $\left(\alpha_{Q}, \alpha_{M}\right)$ polarizabilities. Utilizing the positive sign convention $\nabla \times \mathbf{E}_{\text {inc }}=i c k \mathbf{H}_{\text {inc }}$, we obtain $[28,29]$

$$
\begin{aligned}
& \mathbf{p}=\alpha_{p} \mathbf{E}_{\mathrm{inc}}, \quad \mathbf{m}=\alpha_{m} \mathbf{H}_{\mathrm{inc}}, \\
& \hat{Q}=\alpha_{Q} \frac{\nabla \mathbf{E}_{\mathrm{inc}}+\left(\nabla \mathbf{E}_{\mathrm{inc}}\right)^{T}}{2}, \\
& \hat{M}=\alpha_{M} \frac{\nabla \mathbf{H}_{\mathrm{inc}}+\left(\nabla \mathbf{H}_{\mathrm{inc}}\right)^{T}}{2} .
\end{aligned}
$$

Next, we derive the conditions for the simultaneous suppression of the forward and backward scattering. To do so, we define the angular distribution of the differential scattering cross section by a spherical particle, up to the dipole and quadrupole terms [28]:

$\frac{d \sigma}{d \Omega}(\theta) \cong\left|\frac{\alpha_{p}}{\varepsilon_{0}}+\alpha_{m} \cos \theta+\frac{k^{2}}{4}\left(\frac{\alpha_{Q}}{3 \varepsilon_{0}} \cos \theta+\alpha_{M} \cos 2 \theta\right)\right|^{2}$,

where $\theta$ is the polar angle, and the power distribution is considered to be symmetric in the azimuthal angle plane. Upon inspection of Eq. (3), we find that the coherent scattering from the dipoles is directed mainly in the forward or backward half-spaces depending on whether the term $\operatorname{Re}\left\{\alpha_{p} \alpha_{m}^{*}\right\}$ is positive or negative, respectively [6]. Therefore, to obtain total suppression of the backward scattering $|\theta| \geq 90^{\circ}$ (see Fig. 1), the dipoles have to be in phase and have the same polarizabilities. For suppression of the forward scattering $\left(|\theta| \leq 90^{\circ}\right)$, the dipoles should also have the same polarizabilities; however the phase should be shifted by $\pi$ (the out-of-phase case). These cases are known as the Kerker and anti-Kerker conditions [6]. The former can be formulated as

$$
\left|\alpha_{p}\right|=\left|\varepsilon_{0} \alpha_{m}\right|, \quad \arg \left(\alpha_{p}\right)=\arg \left(\alpha_{m}\right)+2 \pi n,
$$

where $n$ is an integer. For quadrupole only coherent scattering, similar to the case of dipoles, the term $\operatorname{Re}\left\{\alpha_{Q} \alpha_{M}^{*}\right\}$ governs asymmetry of the scattering, but with side lobes as shown in Fig. 1, and they can be called "quadrupolar Kerker conditions" [4,11],

$$
\left|\alpha_{Q}\right|=\left|3 \varepsilon_{0} \alpha_{M}\right|, \quad \arg \left(\alpha_{Q}\right)=\arg \left(\alpha_{M}\right)+2 \pi n,
$$

where $n$ is an integer. Finally, dipole-quadrupole overlapping is defined by the coupling term $\operatorname{Re}\left\{\left(\alpha_{p}+\varepsilon_{0} \alpha_{m}\right) \times\right.$ $\left.\left(\alpha_{Q}+3 \varepsilon_{0} \alpha_{M}\right)^{*}\right\}$. In a particular scattering scenario, if the conditions Eqs. (4) and (5) are satisfied simultaneously, two scattering patterns can be realized depending on the phase relation between the dipoles and quadrupoles, while considering their equal or comparable amplitudes:

$$
\begin{aligned}
\left|\alpha_{p}+\varepsilon_{0} \alpha_{m}\right| & =\frac{k^{2}}{12}\left|\alpha_{Q}+3 \varepsilon_{0} \alpha_{M}\right|, \\
\arg \left(\alpha_{p}+\varepsilon_{0} \alpha_{m}\right) & = \pm \arg \left(\alpha_{Q}+3 \varepsilon_{0} \alpha_{M}\right) .
\end{aligned}
$$

The first condition (with "plus") corresponds to the constructive interference of the dipoles and quadrupoles with enhanced directivity in the forward direction (see, e.g., Ref. [11]). The second scattering regime described by Eq. (6) (with "minus") corresponds to the case when destructive interference between the combined coherent dipoles and combined coherent quadrupoles nearly 
suppresses both forward and backward scattering. This kind of interaction leads to the formation of the transverse scattering pattern, as shown in Fig. 1. The side lobes of the quadrupoles form a scattering pattern in the lateral plane. However, for realistic subwavelength particles, it is impossible to achieve full cancellation because of the optical theorem that links the total extinction cross section of a particle to the forward-scattered fields. Therefore, although forward scattering cannot be completely eliminated, it can be suppressed significantly if the conditions Eqs. (4)-(6) are satisfied. Figure 1(b) presents this concept schematically.

Moreover, we can generalize the conditions Eqs. (4)-(6) to the whole set of multipoles, referring to them as the generalized Kerker and anti-Kerker conditions, respectively $[4,10]$ :

$$
\frac{d \sigma^{T}}{d \Omega}\left(\theta=180^{\circ}\right)=0, \quad \frac{d \sigma^{T}}{d \Omega}\left(\theta=0^{\circ}\right) \cong 0
$$

The normalized differential scattering cross sections for a spherical Si particle of $120 \mathrm{~nm}$ radius are shown in Fig. 2, where we employ the Mie theory and experimental data [30] to solve Eq. (3) for the multipole contributions up to the magnetic quadrupole, and compare it with a sum of all multipole contributions. The conditions Eqs. (4)-(7) are almost satisfied at the wavelength of $609 \mathrm{~nm}$ (size parameter 1.23), when the backward intensity vanishes and the forward scattering intensity reaches its minimum. The result in Fig. 2 proves that the contribution of the higherorder multipoles to the scattered field is negligible and, thus, the dipole-quadrupole approximation remains valid.

Experimental results. - Based on Maxwell equations, we verify our concept by measuring the extinction cross section and scattering patterns of a spherical particle at the (a)

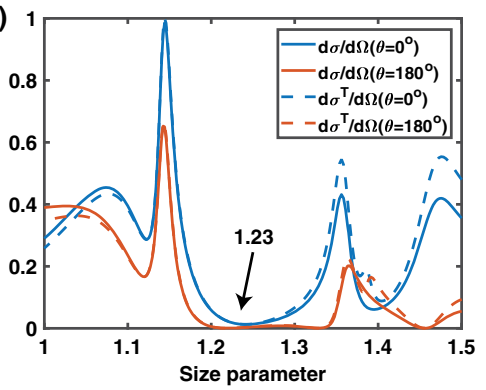

(b)

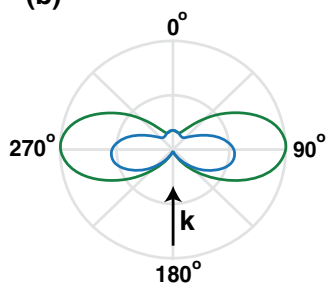

FIG. 2. (a) Normalized differential scattering cross sections calculated with the Mie theory for a silicon sphere of 120-nm radius in free space as functions of the size parameter $=k \times$ radius. The solid lines are calculations for the dipolequadrupolar sphere $[d \sigma / d \Omega$, Eq. (3)] while the dashed lines describe the total set of multipoles $\left[d \sigma^{T} / d \Omega\right.$, Eq. (7)]. In the legend, $\theta=0^{\circ}$ refers the forward direction, and $\theta=180^{\circ}$ to the backward direction. (b) Transverse scattering patterns at 609 -nm wavelength with permittivity $15.44+0.196 i(1.23$ size parameter) [30]: the green (blue) line corresponds to the plane of the incident electric (magnetic) field polarization. microwave frequency range. To mimic the scattering properties of silicon nanoparticles at microwaves, we use $\mathrm{MgO}-\mathrm{TiO}_{2}$ ceramic spheres characterized by the permittivity $\varepsilon=16+0.0179 i$, measured in the range $9-12 \mathrm{GHz}$ [31]. A spherical particle with radius $a=7.5 \mathrm{~mm}$ is located in a microwave chamber for the measurements in the frequency range $4-10 \mathrm{GHz}$. To approximate a plane wave excitation, we use a rectangular horn antenna connected to the transmitting port of a vector network analyzer (Agilent E8362C). The ceramic sphere is located in the far field of the antenna, at the distance of approximately $2.5 \mathrm{~m}$, and the second horn antenna is used as a receiver to observe the transmitted signal. Forward scattering is obtained from the measured transmission coefficient. The total extinction cross section is extracted from the measured complex magnitude of the forward-scattered signal by means of the optical theorem [32]. The measured extinction is compared with the theoretically obtained results (Mie theory) in Fig. 3(a). To measure the two-dimensional scattering diagram, we change slightly the experimental setup. The transmitting antenna and the position of the ceramic sphere remain fixed, whereas the receiving antenna is moved around the spherical particle in the $x-z$ plane. The scattering cross-section patterns for both theoretical (Mie theory) and measured data are shown in Fig. 3(b). We observe the lateral scattering to occur for the broadband off-resonance region from $f=7.6 \mathrm{GHz}$ to $f=7.9 \mathrm{GHz}$.

Nonspherical nanoparticles.-Next, we extend our results to a more general case of nonspherical particles by employing the Cartesian multipole moments $[33,34]$. After applying the procedure analogous to that described above, the conditions for the transverse scattering under $x$-polarized plane wave illumination can be written in the following form [35]:

$$
c p_{x} / m_{y}=1, \quad c Q_{x z} / 3 M_{y z}=1, \quad 2 i c p_{x} / k M_{y z} \cong-1
$$
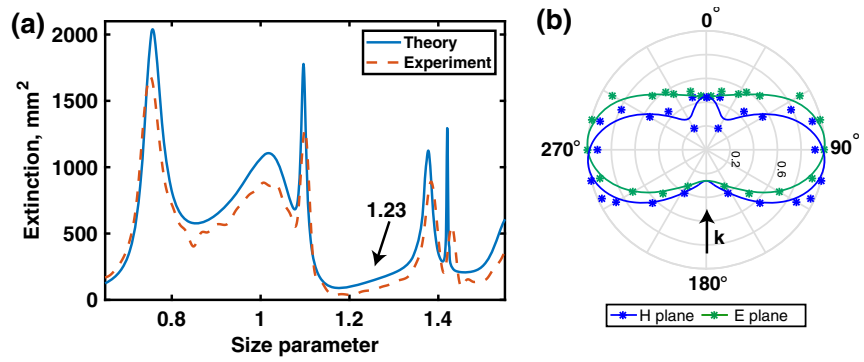

FIG. 3. (a) Comparison of the extinction cross section calculated with the Mie theory and measured experimentally. (b) Scattering patterns at the frequency $f=7.85 \mathrm{GHz}$ ( 1.23 size parameter), where the green (blue) curve corresponds to the plane of the incident electric (magnetic) field polarization. Results are obtained for the ceramic sphere with permittivity $\varepsilon=16+0.0179 i$ and radius $7.5 \mathrm{~mm}$. 
We notice that [3] a lateral-like scattering was revealed as an incidental finding in the study of scattering of Si nanoparticles. However, Ref. [3] provided neither systematic study nor a detailed physical explanation. Figure 4(a) shows the calculated absolute values of the ratios from Eq. (8) and their phases being the phase differences between the involved multipoles. In the wavelength range $700-820 \mathrm{~nm}$, the electric dipole has a sharp Fano profile [47] while the magnetic dipole increases monotonically; therefore the dipoles have the form of two crossing lines. On the other hand, for the wavelength range $\lambda \geq 780 \mathrm{~nm}$, the quadrupole ratio shows linear behavior with constant zero-phase difference. This behavior is actually associated with the longwavelength regime of the quadrupoles. At the particular wavelength $\lambda=788 \mathrm{~nm}$, which is a shared point for the dipole's ratio Fano profile and the quadrupole's longwavelength region (black arrows), one can see that the dipoles are in phase (solid red line) with the nearly equal amplitudes (solid blue line) indicating the Kerker effect. At the same time, the quadrupoles have comparable values, $c Q_{x z} / 3 M_{y z}=0.94$ (dashed blue curve), and are in phase (dashed red curve), which explains the generalized Kerker effect working on the assumption that higher multipoles are negligible. The phase difference between the coherent dipoles and quadrupoles (dotted red line) is about $0.75 \pi$. The corresponding scattering patterns are shown in
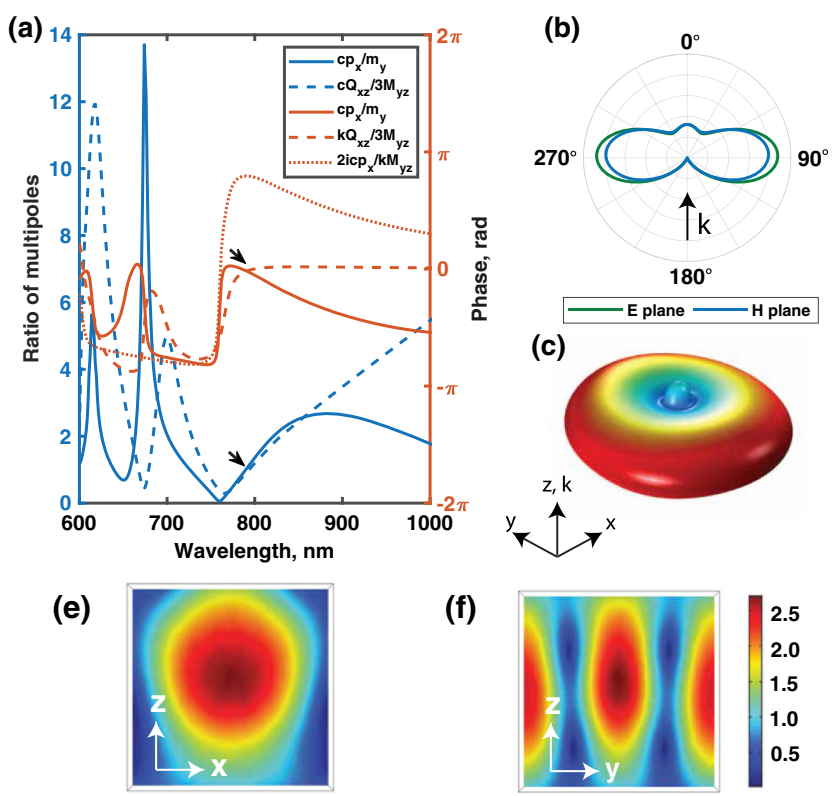

FIG. 4. (a) Amplitudes and phases of the multipole ratios from Eq. (8) for a cubic Si nanoparticle with the edge of $250 \mathrm{~nm}$. The arrows show the wavelength corresponding to the transverse scattering $\lambda=788 \mathrm{~nm}$. (b),(c) Two- and three-dimensional scattering patterns at $\lambda=788 \mathrm{~nm}$. (e),(f) Calculated electric field inside the nanoparticle with the transverse scattering pattern $\lambda=788 \mathrm{~nm}$ in the $x y$ and $x z$ planes, respectively. All calculations were conducted by COMSOL MULTIPHYSIC [23] and the silicon permittivity from Ref. [30].
Figs. 4(b) and 4(c). Hence, we observe almost complete scattering suppression in the forward and backward directions. However, the suppression of the forward scattering is not complete owing to the optical theorem. As a result, we now uncover the physical requirements for the transverse scattering along with the mathematical conditions Eq. (8): the Fano interference of a dipole mode and off-resonant, long-wavelength quadrupoles. These mechanisms provide the necessary phases and amplitudes of the scattered fields in order to obtain the required optical signature. In Supplemental Material [35], we develop this approach further and take into account the presence of a substrate.

In Figs. 4(e) and 4(f), we demonstrate the strong electric field concentration in both the $y-z$ and $x-z$ planes inside the nanoparticle at the wavelength of the transverse scattering. The observed near-field enhancement is accompanied by the strong scattering suppression resembling the case of the anapole mode [24] where the dipole radiation is almost canceled by the field of the electric toroidal moment. This effect of the localization of light could be of a great interest for nonlinear nanophotonics.

Metasurfaces.-Finally, we study a novel type of metasurfaces composed of cubic nanoparticles which allow us to satisfy the condition Eq. (8) for transverse scattering in the visible frequency range. Figure 5(a) shows schematically a square lattice of identical cubic nanoparticles illuminated with a normally incident wave. In the far-field region, the reflection is determined by the effective polarizabilities and multipole moments of a central nanoparticle [48], taking into account the interaction with all other nanoparticles of the metasurface. However, this multipole description can be applied independently to the metasurface with varying lattice spacing [48], which suggests that the obtained conditions Eq. (8) hold.
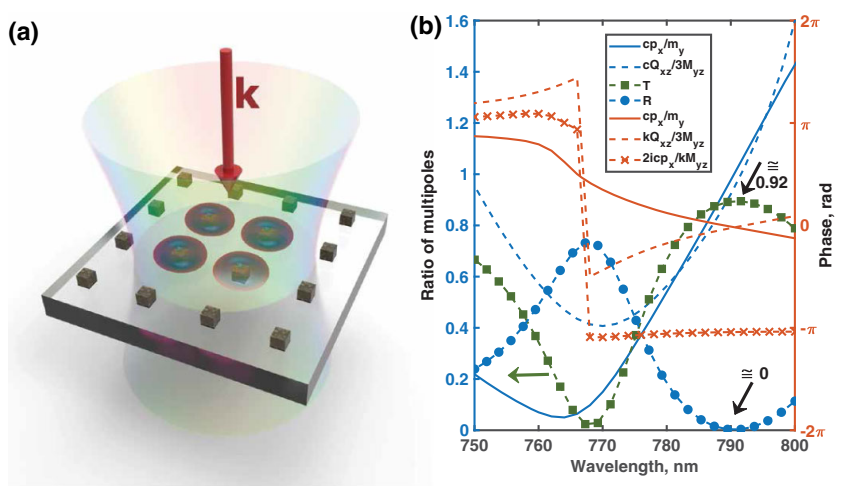

FIG. 5. (a) Schematics of a metasurface being a lattice of cubic nanoparticles with strong transverse scattering. The substrate and the Gaussian beam are shown for better visibility; numerically we consider the metasurface in free space irradiated with a plane wave. (b) Amplitudes (blue) and phases (red) of the multipole ratios defined by Eq. (8) for Si cube with the edge of $250 \mathrm{~nm}$ and the lattice spacing $400 \mathrm{~nm}$, and the corresponding reflection $(R)$ and transmission $(T)$ coefficients. Note that the reflection and transmission coefficients are plotted in accordance with the left $y$ axis. 
We perform full-wave numerical simulations of metasurfaces with the lattice spacing $400 \mathrm{~nm}$. Figure 5(b) shows the ratios of multipoles Eq. (8) in the case of a metasurface, similar to those shown in Fig. 4 for an isolated particle. The polarizabilities are affected by coupling, but the multipole moments within the array still satisfy the transverse scattering conditions Eq. (8). In the wavelength range of $787-793 \mathrm{~nm}$, the coherent dipoles are nearly in phase (solid red curve), and the quadrupoles are also in phase (dashed red curve), but they are out of phase with each other (red curve marked with " $x$ ". With the close-to-unity amplitude ratios (solid and dashed blue curves), the metasurface demonstrates a nearly perfect simultaneous suppression of forward and backward scattering, similar to an isolated nanoparticle (see Fig. 4). Therefore, in this wavelength range, the metasurface reflection vanishes (blue dashed curve labeled with circles), despite the fact that light interacts with the structure providing strong near fields [see Figs. 4(e) and 4(f)], and the transmission (green dashed curve labeled with cubes) is near unity and $100 \%$ transmission can be obtained for the lossless case. In difference to Huygens' surfaces [13], nanoparticles in the metasurfaces introduced here do not scatter in both forward and backward directions. Consequently, an incident wave becomes completely unperturbed. For more information about the impact of the lattice spacing and the presence of the substrate on the invisibility effect, see Ref. [49] and the Supplemental Material [35].

Conclusions. - We have demonstrated a novel effect of the transverse scattering of light by Mie-resonant subwavelength particles with simultaneous suppression of both forward and backward scattering. This generalized Kerker effect occurs when in-phase electric and magnetic dipoles become out of phase with the corresponding quadrupoles. We have obtained the general conditions for the simultaneous suppression of scattering in both forward and backward directions, and have generalized these conditions to nonspherial particles. We have revealed the crucial role played by the electric-dipole Fano resonance together with the off-resonant quadrupoles for achieving transverse scattering, and we have verified our concept in a proof-ofprinciples microwave experiment, with good agreement with analytical and numerical results. Finally, we have studied the metasurfaces composed of the nanoparticles with the transverse scattering patterns and have demonstrated their periodicity-dependent zero reflection. In sharp contrast to Huygens metasurfaces, these novel metasurfaces scatter neither forward nor backward, being almost invisible. The predicted effects can be utilized for efficient beam control, strong field enhancement required for nonlinear interaction, and highly efficient sensing.

The authors acknowledge financial support from the Russian Foundation for Basic Research (Grants No. 1802-00414 and No. 18-52-00005), the Ministry of Education and Science of the Russian Federation (Grant
No. 3.4982.2017/6.7), Israeli Innovation Authority-Kamin Program, Grant No. 62045 (Year 2), and the Strategic Fund of the Australian National University. Experimental characterization of the high-index structures as well as investigation of anapole states were financially supported by Russian Science Foundation (Grants No. 17-79-20379 and No. 17-72-10230, respectively). A. B. E. also acknowledges support from the Deutsche Forschungsgemeinschaft (DFG) within the Cluster of Excellence PhoenixD (EXC 2122, Project ID 390833453). Y. K. thanks Wei Liu for useful discussions and suggestions.

[1] S. Kruk and Y. Kivshar, Functional meta-optics and nanophotonics governed by Mie resonances, ACS Photonics 4, 2638 (2017).

[2] Y. Kivshar, All-dielectric meta-optics and non-linear nanophotonics, Natl. Sci. Rev. 5, 144 (2018).

[3] P. D. Terekhov, K. V. Baryshnikova, Y. A. Artemyev, A. Karabchevsky, A. S. Shalin, and A. B. Evlyukhin, Multipolar response of nonspherical silicon nanoparticles in the visible and near-infrared spectral ranges, Phys. Rev. B 96, 035443 (2017).

[4] W. Liu and Y.S. Kivshar, Generalized Kerker effects in nanophotonics and meta-optics, Opt. Express 26, 13085 (2018).

[5] M. Kerker, D.-S. Wang, and C. L. Giles, Electromagnetic scattering by magnetic spheres, J. Opt. Soc. Am. 73, 765 (1983).

[6] M. Nieto-Vesperinas, R. Gomez-Medina, and J. J. Saenz, Angle-suppressed scattering and optical forces on submicrometer dielectric particles, J. Opt. Soc. Am. A 28, 54 (2011).

[7] A. E. Krasnok, A. E. Miroshnichenko, P. A. Belov, and Y. S. Kivshar, Huygens optical elements and Yagi-Uda nanoantennas based on dielectric nanoparticles, JETP Lett. 94, 593 (2011).

[8] P. D. Terekhov, K. V. Baryshnikova, A. S. Shalin, A. Karabchevsky, and A.B. Evlyukhin, Resonant forward scattering of light by high-refractive-index dielectric nanoparticles with toroidal dipole contribution, Opt. Lett. 42, 835 (2017).

[9] M. Dubois, L. Leroi, Z. Raolison, R. Abdeddaim, T. Antonakakis, J. de Rosny, A. Vignaud, P. Sabouroux, E. Georget, B. Larrat, G. Tayeb, N. Bonod, A. Amadon, F. Mauconduit, C. Poupon, D. Le Bihan, and S. Enoch, Kerker Effect in Ultrahigh-Field Magnetic Resonance Imaging, Phys. Rev. X 8, 031083 (2018).

[10] W. Liu, J. Zhang, B. Lei, H. Ma, W. Xie, and H. Hu, Ultra-directional forward scattering by individual core-shell nanoparticles, Opt. Express 22, 16178 (2014).

[11] A. Pors, S. K. H. Andersen, and S. I. Bozhevolnyi, Unidirectional scattering by nanoparticles near substrates: Generalized Kerker conditions, Opt. Express 23, 28808 (2015).

[12] M. F. Picardi, A. V. Zayats, and F. J. Rodríguez-Fortuño, Janus and Huygens Dipoles: Near-Field Directionality Beyond Spin-Momentum Locking, Phys. Rev. Lett. 120, 117402 (2018).

[13] M. Decker, I. Staude, M. Falkner, J. Dominguez, D. N. Neshev, I. Brener, T. Pertsch, and Y.S. Kivshar, 
High-efficiency dielectric Huygens surfaces, Adv. Opt. Mater. 3, 813 (2015).

[14] S. Kruk, B. Hopkins, I. I. Kravchenko, A. Miroshnichenko, D. N. Neshev, and Y. S. Kivshar, Broadband highly efficient dielectric metadevices for polarization control, APL Photonics 1, 030801 (2016).

[15] P. Genevet, F. Capasso, F. Aieta, M. Khorasaninejad, and R. Devlin, Recent advances in planar optics: From plasmonic to dielectric metasurfaces, Optica 4, 139 (2017).

[16] I. Staude, T. Pertsch, and Y. S. Kivshar, All-dielectric resonant meta-optics lightens up, ACS Photonics 6, 802 (2019).

[17] P. D. Terekhov, V. E. Babicheva, K. V Baryshnikova, A. S. Shalin, A. Karabchevsky, and A. B. Evlyukhin, Multipole analysis of dielectric metasurfaces composed of nonspherical nanoparticles and lattice invisibility effect, Phys. Rev. B 99, 045424 (2019).

[18] J. Li, N. Verellen, D. Vercruysse, T. Bearda, L. Lagae, and P. Van Dorpe, All-dielectric antenna wavelength router with bidirectional scattering of visible light, Nano Lett. 16, 4396 (2016).

[19] G. Lu, Y. Wang, R. Y. Chou, H. Shen, Y. He, Y. Cheng, and Q. Gong, Directional side scattering of light by a single plasmonic trimer, Laser Photonics Rev. 9, 530 (2015).

[20] Y.X. Ni, L. Gao, A. E. Miroshnichenko, and C. W. Qiu, Controlling light scattering and polarization by spherical particles with radial anisotropy, Opt. Express 21, 8091 (2013).

[21] J. Y. Lee, A. E. Miroshnichenko, and R.-K. Lee, Simultaneously nearly zero forward and nearly zero backward scattering objects, Opt. Express 26, 30393 (2018).

[22] O. Peňa and U. Pal, Scattering of electromagnetic radiation by a multilayered sphere, Comput. Phys. Commun. 180, 2348 (2009).

[23] COMSOL Multiphysics®, www.comsol.com.

[24] K. V. Baryshnikova, D. A. Smirnova, B. S. Luk'yanchuk, and Y.S. Kivshar, Optical anapoles: Concepts and applications, Adv. Opt. Mater., https://doi.org/10.1002/adom .201801350 (2019).

[25] K. Koshelev, G. Favraud, A. Bogdanov, Y. Kivshar, and A. Fratalocchi, Nonradiating photonics with resonant dielectric nanostructures, Nanophotonics, https://doi.org/10.1515/ nanoph-2019-0024 (2019).

[26] G. Grinblat, Y. Li, M. P. Nielsen, R. F. Oulton, and S. A. Maier, Degenerate four-wave mixing in a multiresonant Germanium nanodisk, ACS Photonics 4, 2144 (2017).

[27] L. Carletti, K. Koshelev, C. De Angelis, and Y. Kivshar, Giant Nonlinear Response at the Nanoscale Driven by Bound States in the Continuum, Phys. Rev. Lett. 121, 033903 (2018).

[28] J. D. Jackson, Classical Electrodynamics 3rd ed. (Wiley, New York, 1998).

[29] A. Alú and N. Engheta, Guided propagation along quadrupolar chains of plasmonic nanoparticles, Phys. Rev. B 79, 235412 (2009).

[30] D. E. Aspnes and A. A. Studna, Dielectric functions and optical parameters of $\mathrm{Si}, \mathrm{Ge}, \mathrm{GaP}, \mathrm{GaAs}, \mathrm{GaSb}, \mathrm{InP}, \mathrm{InAs}$, and InSb from 1.5 to $6.0 \mathrm{eV}$, Phys. Rev. B 27, 985 (1983).

[31] A. Kanareykin, W. Gai, E. Nenasheva, and A. Altmark, Transformer ratio enhancement experiment, in Proc. 2003 Bipolar/BiCMOS Circuits Technol. Meet. (IEEE Cat. No.03CH37440), edited by J. Chew, P. Lucas, and
S. Webber (IEEE, Portland, 2003), pp. 1894-1896, https://doi.org/10.1109/PAC.2003.1288711.

[32] R. G. Newton, Optical theorem and beyond, Am. J. Phys. 44, 639 (1976).

[33] R.E. Raab and O. L. De Lange, Multipole Theory in Electromagnetism: Classical, Quantum, and Symmetry Aspects, with Applications (Oxford University Press, New York, 2005).

[34] C. Vrejoiu, Electromagnetic multipoles in Cartesian coordinates, J. Phys. A 35, 9911 (2002).

[35] See Supplemental Material at http://link.aps.org/ supplemental/10.1103/PhysRevLett.122.193905 for details of the calculations, which includes Refs. [36-46].

[36] A. B. Evlyukhin, T. Fischer, C. Reinhardt, and B. Chichkov, Optical theorem and multipole scattering of light by arbitrary shaped nanoparticles, Phys. Rev. B 94, 205434 (2016).

[37] D. R. Bohren and C. F. Huffman, Absorption and Scattering of Light by Small Particles (Wiley, New York, 1983).

[38] S. Q. Li and K. B. Crozier, Origin of the anapole condition as revealed by a simple expansion beyond the toroidal multipole, Phys. Rev. B 97, 245423 (2018).

[39] L. Wei, Z. Xi, N. Bhattacharya, and H. P. Urbach, Excitation of the radiationless anapole mode, Optica 3, 799 (2016).

[40] A. E. Miroshnichenko, A. B. Evlyukhin, Y. S. Kivshar, and B. N. Chichkov, Substrate-induced resonant magnetoelectric effects for dielectric nanoparticles, ACS Photonics 2, 1423 (2015).

[41] L. Novotny and B. Hecht, Principles of Nano-Optics (Cambridge University Press, Cambridge, England, 2006).

[42] V. E. Babicheva and A. B. Evlyukhin, Resonant lattice Kerker effect in metasurfaces with electric and magnetic optical responses, Laser Photonics Rev. 11, 1700132 (2017).

[43] A. S. Shalin, Broadband blooming of a medium modified by an incorporated layer of nanocavities, JETP Lett. 91, 636 (2010).

[44] C. R. Simovski, A. S. Shalin, P. M. Voroshilov, and P. A. Belov, Photovoltaic absorption enhancement in thin-film solar cells by non-resonant beam collimation by submicron dielectric particles, J. Appl. Phys. 114, 103104 (2013).

[45] P. M. Voroshilov, C. R. Simovski, P. A. Belov, and A. S. Shalin, Light-trapping and antireflective coatings for amorphous Si-based thin film solar cells, J. Appl. Phys. 117, 203101 (2015).

[46] D. A. Baranov, P. A. Dmitriev, I. S. Mukhin, A. K. Samusev, P. A. Belov, C. R. Simovski, and A. S. Shalin, Broadband antireflective coatings based on two-dimensional arrays of subwavelength nanopores, Appl. Phys. Lett. 106, 171913 (2015).

[47] M. F. Limonov, M. V. Rybin, A. N. Poddubny, and Y. S. Kivshar, Fano resonances in photonics, Nat. Photonics 11, 543 (2017).

[48] A. B. Evlyukhin, C. Reinhardt, A. Seidel, B. S. Lukyanchuk, and B.N. Chichkov, Optical response features of Sinanoparticle arrays, Phys. Rev. B 82, 045404 (2010).

[49] Increasing the lattice spacing leads to a broadening of the invisibility region. The interparticle coupling becomes stronger and, as a result, the multipole resonances experience an incoherent shift of the spectrum. On the other hand, a substrate introduced to the metasurface will only shift the near-zero region to shorter wavelengths. 